\title{
Relato de experiência de grupo de pesquisa em epidemiologia e cardiologia
}

\author{
Experience report from an epidemiology and cardiology research group \\ Lucas Soares Bezerra ${ }^{1,2}$ (D), Marcelo Antônio Oliveira Santos-Veloso ${ }^{1,3}$ (D), Ândrea Virgínia Chaves-Markman ${ }^{1,2,4}$ (D), \\ Andrea Bezerra de Melo da Silveira Lordsleem ${ }^{2,4}$ (D), Sandro Gonçalves de Lima ${ }^{2,4}$ (iD)
}

\begin{abstract}
RESUMO
A formação de grupos acadêmicos durante o curso de medicina é importante para a construção e compartilhamento de conhecimentos e habilidades fundamentais para a prática médica. O presente trabalho relata a experiência de um ano de atividades (2017-2018) de um grupo de pesquisa em epidemiologia e cardiologia. Apresentamos os principais pontos de motivação, objetivos e funcionamento do grupo, no intuito de fornecer um modelo estrutural que possa servir de inspiração e debate para a formação de outros grupos de pesquisa constituídos por estudantes de medicina ao redor do país. Ao longo de um ano foram apresentados em eventos científicos os resultados das pesquisas do grupo, além da publicação de artigos em periódicos científicos na área da saúde, totalizando 12 trabalhos acadêmicos. Como meio de fornecer uma ferramenta de avaliação quantitativa de análise das atividades do grupo, foram apresentados os resultados da autoavaliação desenvolvida pelos membros acadêmicos após um ano de atividades. Considerando o elevado número de publicações biomédicas e o aumento na exigência de conhecimentos em pesquisa e medicina baseada em evidências nos últimos anos, a formação de grupos de pesquisa apresenta-se como uma alternativa para complementação de conhecimentos teóricos e práticos para a formação médica, permitindo aos futuros profissionais uma visão crítica da produção do conhecimento científico, uma prática médica baseada em evidências, um melhor enfretamento das exigências de mercado e dos interesses econômicos na área de saúde.
\end{abstract}

Palavras-chave: Educação Médica; Pesquisa; Cardiologia; Epidemiologia.

\begin{abstract}
The establishment of academic groups during medical school is important for the construction and sharing of knowledge and fundamental skills to good medical practice. This paper reports a one-year experience of activities (2017-2018) of a research group in epidemiology and cardiology. The presentation of the motivation reasons, objectives, and performance of the group to provide a structural model that could serve as inspiration and debate for the establishment of other research groups made up from and for medical students from all over the world. During one year of activity, the findings of the group research were presented in scientific events, as well as the publication of articles in scientific journals in field of health sciences, totaling 12 academic papers. As a means of providing a quantitative evaluation tool for the analysis of the group's activities, the results of the self-assessment developed by the academic members after one year of activities were presented. Considering the high number of biomedical publications and the increase in the demand for knowledge in research and evidence-based medicine in recent years, the establishment of research groups is an alternative to complement theoretical and practical knowledge for medical education, allowing to future professionals a critical view of the production of scientific knowledge, evidence-based medical practice, a better understanding of market demands, and economic interests in health.
\end{abstract}

Keywords: Education, Medical; Research; Cardiology; Epidemiology.

1. Programa de Pós-graduação em Inovação Terapêutica, Centro de Biociências da Universidade Federal de Pernambuco (UFPE), Recife (PE), Brasil.

2. Curso de Medicina, Centro Universitário Maurício de Nassau (UNINASSAU), Recife (PE), Brasil.

3. Programa de Residência em Clínica Médica, Hospital dos Servidores do Estado de Pernambuco, Recife (PE), Brasil

4. Departamento de Cardiologia, Hospital das Clínicas da Universidade Federal de Pernambuco (HC/UFPE), Recife (PE), Brasil.

$\bowtie$ Lucas Soares Bezerra. $1^{\circ}$ andar do prédio do Centro de Biociências - CB, UFPE. Av. Prof. Moraes Rego, S/N - Cidade Universitária. CEP: 50670-420. Recife (PE), Brasil. lucassbezerra@gmail.com | Recebido em: 02/09/2019 | Aprovado em: 08/11/2019 


\section{INTRODUÇÃO}

O Grupo de Pesquisa em Epidemiologia e Cardiologia (EPICARDIO) é um grupo de pesquisa cadastrado no ano de 2017 na plataforma SIGProj (protocolo 278797.1384.265895.25072017) da Universidade Federal de Pernambuco - UFPE. Sua logo está representada na Figura 1.

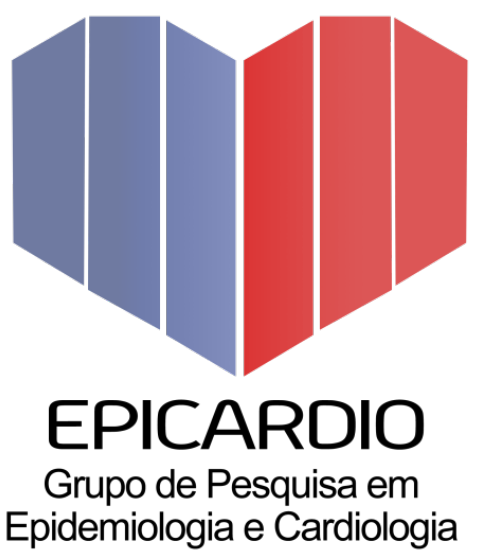

Figura 1: Logo do Grupo de Pesquisa em Epidemiologia e Cardiologia. O coração é formado por barras verticais que lembram gráficos. As cores se relacionam às representações do sangue arterial e venoso. O termo EPICARDIO é formado pela junção das palavras "EPIdemiologia" e "CARDIOlogia".

Um dos principais intuitos com a formação do grupo foi trazer uma nova perspectiva no meio acadêmico, uma vez que maioria dos grupos estudantis nas escolas médicas é formado pelas Ligas Acadêmicas, projetos que possuem enfoque em extensão, não em pesquisa. Além delas, são frequentes outros projetos de extensão que promovem atividades de cunho social e/ou de ensino, mas com baixa produção científica ${ }^{1}$.

A ideia inicial do grupo foi a de fomentar o interesse e o desenvolvimento de pesquisas por parte dos estudantes de Medicina. Tais projetos seguiram com foco na área de Cardiologia e Epidemiologia, em concordância com a formação de coordenador e orientadores.

O foco do presente trabalho é apresentar um relato de experiência das atividades do grupo entre os anos 2017 a 2018, com a finalidade de compartilhar expectativas e modelos de educação em pesquisa que possam servir de inspiração na formação de outros grupos ao redor do país.

\section{A importância da pesquisa na educação médica}

Com o avanço nos meios de comunicação e de acesso à informação, tem se tornado cada vez mais necessário aos estudantes e profissionais médicos aprender a buscar e a interpretar o conteúdo de pesquisas científicas. A Medicina Baseada em Evidências (MBE) representa a importância do conhecimento científico sobre formas de conhecimento baseadas em experiência profissional enquanto guia de condutas clínicas.

Os grupos de pesquisa têm o papel de, a partir do conhecimento tecnológico e científico, traçar uma ponte entre a sociedade e suas necessidades e a pesquisa científica ${ }^{1,2}$. Isso significa compreender que o sujeito da pesquisa demanda, ele próprio, colher os frutos do investimento científico, os quais os pesquisadores se propõem a fazer. Vivenciar a realidade do que se estuda permite criar um vínculo entre teoria e prática, além de promover a formação de futuros profissionais mais críticos e engajados em oferecer mudanças ao sistema de saúde ${ }^{3,4}$.

Além disso, tem sido possível perceber o grande volume de estudos publicados no campo da saúde, porém, com muitos dados não confiáveis. Estima-se que apenas $5 \%$ dos artigos científicos publicados na área sejam reproduzíveis ${ }^{5}$. Diante disso, uma postura crítica por parte dos estudantes e profissionais dessa área é de extrema necessidade.

Percebemos que o processo de aprendizagem do EPICARDIO segue as cinco etapas apresentadas pelo Grupo para Recursos em Medicina Baseada em Evidências da Universidade McMaster: definição do problema, busca em bases de dados, avaliação crítica das informações, aplicação da informação ao paciente e por último a avaliação eficaz desta aplicação ${ }^{6}$. Este feedback ao fim permite compreender a aplicabilidade e dimensões da pesquisa realizada.

Ao longo do curso médico o estudante é frequentemente submetido a diferentes tipos de testes e avaliações de habilidades e conhecimento técnico7. Participar do processo de formação de todas as etapas anteriormente citadas permite ao estudante entender as engrenagens do processo de produção científica, o que leva a um melhor 
entendimento e visão crítica dos estudos que são publicados no meio acadêmico, bem como de entender as suas aplicabilidades.

Todas essas demandas seguem as principais recomendações mundiais da educação médica, como a Declaração de Alma Ata e o Projeto Educação Médica nas Américas, fortalecendo as mudanças que têm sido aplicadas na estrutura curricular dos cursos médicos 8,9 .

\section{Formação dos membros}

O EPICARDIO tem sua composição formada por oito membros acadêmicos e quatro orientadores. Dentre os acadêmicos, dois executam a função de Diretores (um geral e um científico), e dentre os orientadores um é o coordenador. Todos os estudantes (sendo seis de instituição privada) são provenientes de cursos de Medicina (de três diferentes instituições), do $3^{\circ}$ ao $10^{\circ}$ período da graduação. Durante o processo seletivo foram recebidas requisições de estudantes de outros cursos de saúde como enfermagem e farmácia para a abertura de vagas também para eles, e tal expansão e integração tornou-se um obje- tivo para as próximas gestões do grupo, quando sua estrutura estivesse mais bem consolidada.

$O$ processo seletivo forneceu seis vagas e foi estruturado em duas fases: teste cognitivo e entrevista, além de análise curricular, realizados com uma semana de intervalo. A primeira fase possuiu peso de $50 \%$, a entrevista $30 \%$ e análise curricular $20 \%$ da nota final.

O teste cognitivo foi composto por 30 questões das quais dez relacionadas à epidemiologia clínica e 20 de: fisiologia cardiovascular aplicada à clínica, bioestatística, medicina baseada em evidências e interpretação de estudos em língua inglesa. O conteúdo do teste buscou focar em pontos de grande importância para o entendimento e desenvolvimento de novos estudos, e por isso incluiu compreensão de produções científicas em língua inglesa. Ao todo 32 estudantes se inscreveram, com uma média de 5,3 candidatos por vaga.

A análise curricular buscou pontuar experiências acadêmicas de ensino, pesquisa e extensão, compreendendo desde monitorias e participações em eventos até conhecimentos em línguas estrangeiras. A listagem com as pontuações, conforme divulgada em edital de seleção, está descrita na Tabela 1.

\section{Tabela 1}

Pontuações da análise curricular do processo seletivo 2017 do EPICARDIO, com valor máximo de 10 pontos.

\begin{tabular}{|c|c|c|}
\hline Atividade & Pontuação individual & Pontuação máxima \\
\hline Monitoria & 1 ponto/semestre & 2 pontos \\
\hline Iniciação Científica & 2 pontos/semestre & 4 pontos \\
\hline Projeto de Extensão & 1 ponto/semestre & 2 pontos \\
\hline Idioma (por idioma) & $\begin{array}{l}\text { Fluente/Avançado }-1 \text { ponto } \\
\text { Intermediário }-0,5 \text { ponto } \\
\text { Básico }-0,25 \text { ponto }\end{array}$ & Ilimitada \\
\hline Voluntariado & 0,5 ponto/semestre & 1 ponto \\
\hline Intercâmbio & 1 ponto/semestre & 2 pontos \\
\hline Participação em eventos científicos & $\begin{array}{l}\text { Internacional - } 1 \text { ponto/evento } \\
\text { Brasileiro - 0,5 ponto/evento } 3 \text { pontos } \\
\text { Regional/Local - 0,25 ponto/evento }\end{array}$ & 3 pontos \\
\hline $\begin{array}{l}\text { Apresentação de trabalhos em } \\
\text { eventos relacionados à cardiologia } \\
\text { ou epidemiologia }\end{array}$ & $\begin{array}{c}\text { Pôster - 0,5 ponto/cada } \\
\text { Apresentação Oral - } 1 \text { ponto/cada }\end{array}$ & 4 pontos \\
\hline $\begin{array}{l}\text { Apresentação de trabalhos em } \\
\text { eventos em outras áreas }\end{array}$ & $\begin{array}{c}\text { Pôster - 0,1 ponto/cada } \\
\text { Apresentação Oral - 0,25 ponto/cada }\end{array}$ & 2 pontos \\
\hline $\begin{array}{l}\text { Resumos publicados na área de } \\
\text { cardiologia ou epidemiologia }\end{array}$ & 1 ponto/resumo & 2 pontos \\
\hline
\end{tabular}

(Continua...) 
Tabela 1 (Continua...)

\begin{tabular}{lcc}
\hline Atividade & Pontuação individual & Pontuação máxima \\
\hline Resumos publicado em outras áreas & 0,5 ponto/resumo & 1 ponto \\
$\begin{array}{l}\text { Publicação de artigos completos em } \\
\text { periódicos }\end{array}$ & Qualis A2-A1 - 2 pontos/artigo & 6 pontos \\
& Qualis B3-B1 - 1 pontos/artigo & \\
Qualis B5-B4 - 0,5 ponto/artigo & 2 pontos \\
Participação em cursos livres & Qualis C - 0,1 ponto/artigo & 2 pontos \\
$\begin{array}{l}\text { Organização de cursos/eventos } \\
\begin{array}{l}\text { Prêmios de reconhecimento acadê- } \\
\text { micos/científico }\end{array}\end{array}$ & Até 19h de CH - 0,25 ponto/curso & 2 pontos \\
\hline
\end{tabular}

Após divulgação dos resultados do processo seletivo, foi realizada uma reunião introdutória na qual os membros foram apresentados uns aos outros, assim como discutidos tópicos gerais acerca do funcionamento do grupo. Um dos pontos abordados foi o modelo de orientação desenvolvido, segundo o qual dois estudantes se tornariam os membros responsáveis pelo desenvolvimento de uma pesquisa-chave (pesquisa principal voltada ao grupo) de um professor orientador. O modelo se encontra representado na Figura 2. O conceito de pesquisa-chave foi utilizado para enfatizar a liberdade de desenvolver outros estudos por parte dos membros, inclusive com divisões diferentes das pré-estabelecidas na reunião inaugural, porém, a cobrança por parte da diretoria seria feita sobre a pesquisa-chave, apenas. A lista de pesquisas definidas encontra-se na Tabela 2.

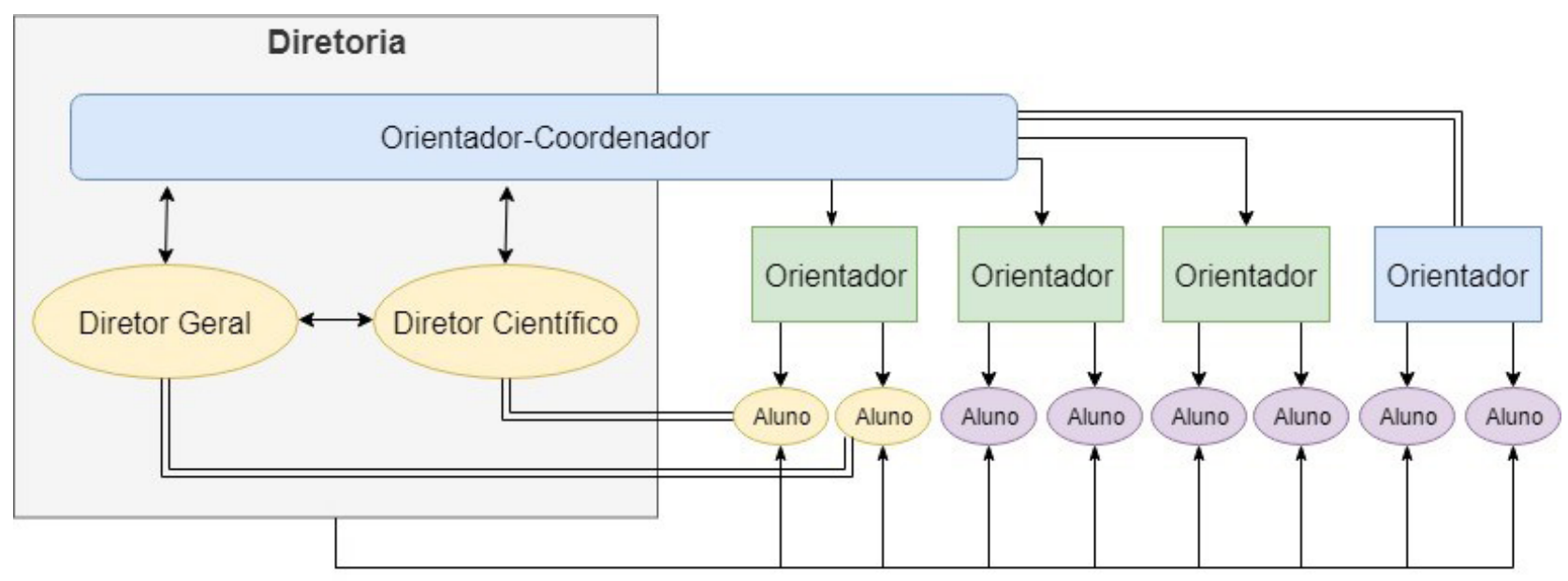

Figura 2: Modelo organizacional do EPICARDIO: a Diretoria é o órgão deliberativo composto por um orientador-coordenador e dois alunos com funções de Diretor Geral e Diretor Científico. O orientador-coordenador gere a equipe de orientadores. Cada orientador teve dois estudantes enquanto pesquisadores responsáveis em sua pesquisa-chave. As atividades de cada aluno eram monitoradas paralelamente pela Diretoria e por seu orientador responsável. 


\section{Tabela 2}

Linhas de pesquisa e temas definidos para estudo durante o ano 2017-2018 de atividades do EPICARDIO.

\begin{tabular}{cll}
\hline Orientador & Linha de pesquisa & Descrição \\
\hline 1 & $\begin{array}{l}\text { Miocardiopatias e doenças } \\
\text { raras }\end{array}$ & $\begin{array}{l}\text { Avaliação de aspectos clínicos, epidemiológicos, fisiopatológicos e mar- } \\
\text { cadores de risco em cardiomiopatias raras, em especial: cardiomiopatia } \\
\text { hipertrófica, doença de Yamaguchi e doença de Fabry. } \\
\text { Avaliação das características cardiovasculares pré e pós paratiroidecto- } \\
\text { mia em pacientes com Doença Renal Crônica dialítica. }\end{array}$ \\
2 & Cardiorrenal & $\begin{array}{l}\text { Metabolismo lipídico e este- } \\
\text { atose hepática } \\
\text { Evidemiologãa, diagnóstico fibrogênese hepática na esteato-hepatite não alcoólica em } \\
\text { obesos submetidos à cirurgia bariátrica }\end{array}$ \\
& $\begin{array}{l}\text { Avaliação dos aspectos epidemiológicos, diagnósticos e terapêuticos da } \\
\text { e tratamento da Hiperten- } \\
\text { são Arterial Sistêmica }\end{array}$ & $\begin{array}{l}\text { Hipertensão Arterial Sistêmica, da Hipertensão Reativa, da Pseudocrise } \\
\text { Hipertensiva, da Hipertensão Mascarada e da Hipertensão Resistente. }\end{array}$ \\
\hline
\end{tabular}

\section{Funcionamento e atividades}

Inicialmente, foi estabelecido que as atividades do grupo constariam basicamente de: desenvolvimento da pesquisa-chave, produção de textos acadêmicos para a seção científica do site do grupo, a organização de um evento acadêmico, apresentações de artigos científicos em reuniões mensais, além da publicação dos resultados das pesquisas em eventos científicos e em periódicos.

A redação dos textos acadêmicos tinha como base realizar a análise crítica de artigos científicos publicados, selecionados à escolha do estudante. Já as reuniões mensais eram compostas de uma fase com apresentação de um artigo científico por um acadêmico (selecionado por um dos orientadores), seguido de discussão entre os membros sempre com a presença de um orientador. $O$ segundo momento das reuniões servia para avaliar o andamento das pesquisas e discutir possíveis dúvidas e dificuldades encontradas.

No segundo semestre de atividades do grupo foi decidido, por solicitação de alguns membros acadêmicos, pela suspensão dos textos da seção científica para o site, assim como da organização do evento acadêmico. Tais medidas tenderiam facilitar a execução das demandas das pesquisas-chave, além de permitir a produção dos materiais finais a serem publicados e/ou apresentados.

A indisponibilidade de tempo sempre foi uma das principais dificuldades apontadas pelos membros para o adequado cumprimento das atividades do grupo, além de limitações de conhecimento teórico e prático prévios para o desenvolvimento de pesquisas.

\section{RESULTADOS OBTIDOS}

Como resultado dos trabalhos desenvolvidos durante o período, houve a apresentação de sete pôsteres em eventos científicos (três das pesquisas chaves mais quatro com participação dos membros), incluindo um trabalho premiado, e também houve a produção de cinco artigos científicos aceitos para publicação em periódicos e um artigo já publicado até esta data.

Ao término das atividades foi realizado um questionário organizado pela diretoria do grupo para avaliação da percepção dos membros acadêmicos acerca das atividades desenvolvidas. Este foi de preenchimento voluntário e anônimo, a partir de formulário padrão disponibilizado apenas aos participantes do projeto. Os resultados estão descritos na Figura 3. 


\section{1) Qual nota você daria para o desempenhop geral do grupo até o momento?}

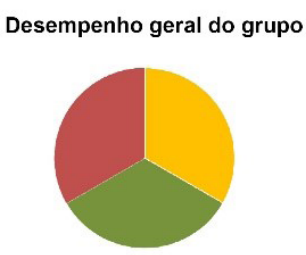

Notas representadas pelas cores:

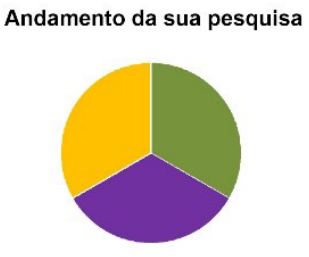

\begin{tabular}{l|l|l|l|l|l}
1 & 2 & 3 & 4 & &
\end{tabular}

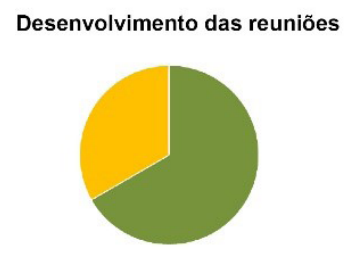

2) Como voce avalia a reunião científica?

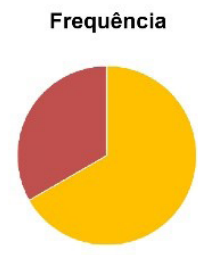

Efetividade das pautas
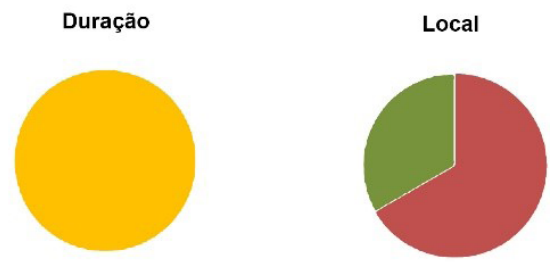

Conceitos representados pelas cores:

Pouco

Regular Suficiente

Ótimo

\section{3) Autoavaliação}

Quanto você cresceu

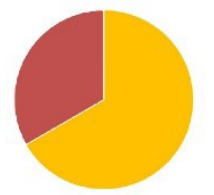

Considera-se bem assistido

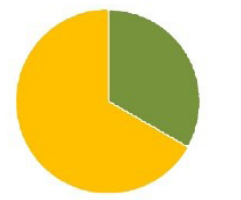

Tem se dedicado

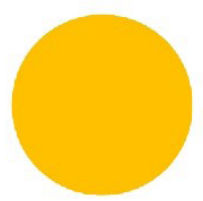

Resultados vs tempo

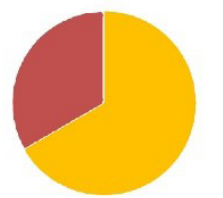

Conceitos representados pelas cores:

Pouco

Regular

Suficiente

Ótimo/Mais que o esperado

Figura 3: Resultados da autoavaliação dos estudantes quanto às atividades e organização do EPICARDIO, assim como de crescimento acadêmico dentre 2017-2018. A questão 1 foi avaliada a partir das notas de 1 a 5 . As questões 2 e 3 foram avaliadas a partir de conceitos, conforme imagem.

\section{ANÁLISE DOS RESULTADOS}

A autoavaliação do desempenho do grupo por seus membros apresentou um padrão heterogêneo de distribuição, com resultados compatíveis com as expectativas da coordenação e da direção do EPICARDIO. Ainda assim, um terço dos participantes pontuou nota 2 para o desempenho global do grupo. Ao confrontar esse resultado com outros pontos, vemos que todos os participantes avaliaram a dedicação individual como suficiente e andamento das pesquisas individuais como regular ou muito bom (notas 3 a 5). A discrepância entre o indicador cole- tivo e individual poderia ser justificada em parte pela superestimativa dos resultados individuais no panorama geral e, por outro lado, porque os estudantes percebem sua participação no desenvolvimento das pesquisas como suficiente, porém, avaliam como insuficiente as demais atividades do grupo.

Atividades burocráticas intrinsecamente ligados ao processo da produção científica poderiam representar um dos principais entraves a desestimular a proximidade do aluno com a pesquisa científica. A taxa de desistência ao longo do ano foi de $25 \%$ e o principal motivo foi tempo insuficiente para dedicar as atividades de pesquisa. 


\section{Desafios}

Os modelos de ensino médico vem sendo alvo de debates recorrentes nos últimos anos. Entretanto, dentre as pautas, a vivência acadêmica ou seja, o contato com mestrandos, doutorandos e pesquisadores - e produção de conhecimento científico tem pouco espaço. Dentre os motivos, podemos citar como principais contribuintes: a baixa motivação do médico e estudante de medicina pela produção científica e a baixa disponibilidade de orientadores qualificados que estimulem a iniciação científica (IC) e interesse pela academia ${ }^{10}$.

Apesar disso, um estudo com alunos de IC de um curso médico mostrou que ao final do $6^{\circ}$ ano somente $7 \%$ dos alunos referiam não ter nenhum interesse em pesquisa ${ }^{11}$. A alta concorrência do mercado médico, especialmente com a abertura de novas escolas de medicina e restrição das vagas de residência, podem ser fatores que tem contribuído para essa nova tendência.

Os novos currículos do curso médico cada vez mais têm implementado disciplinas relacionadas à pesquisa médica, seguindo a tendência dos países desenvolvidos ${ }^{12}$, entretanto, tal inclusão nem sempre parece refletir-se nos indicadores de produção acadêmica na graduação ${ }^{1}$. Sabe-se que médicos com experiência de IC durante o curso possuem maiores chances de formalizar títulos acadêmicos - como mestrado e doutorado -, bem como possuem melhor desempenho do que aqueles que não desenvolveram nenhuma atividade de pesquisa durante o curso..$^{13}$

Na maioria dos países desenvolvidos, é esperado que o médico realize atividades de pesquisa e produção científica, realidade ainda não incorporada no mercado Brasileiro ${ }^{12}$. Entretanto, com a concepção dos complexos econômicos-industriais em saúde e a perspectiva da inter-relação entre desenvolvimento científico-econômico nacional e a qualidade da prestação de serviços em saúde ${ }^{14}$, espera-se uma mudança nesse cenário.

\section{CONSIDERAÇÕES FINAIS}

Diante das novas exigências apresentadas à estrutura curricular dos cursos de medicina e das demandas advindas do aumento no número de publica- ções e desenvolvimento de estudos nas últimas décadas, consideramos importante o aperfeiçoamento dos conhecimentos em pesquisa ao longo do curso médico para uma melhor formação profissional.

A formação de grupos de pesquisa pode ser uma alternativa interessante de imersão prática dos conteúdos teóricos abordados na graduação, que permitirá o desenvolvimento de habilidades tanto para a avaliação crítica dos estudos que vem sendo publicados como para o próprio desenvolvimento de pesquisas, com impacto tanto na formação acadêmica individual quanto ao estreitar laços da pesquisa científica com as necessidades da sociedade.

\section{CONFLITO DE INTERESSES}

Não existem conflitos de interesse. Nenhum financiamento foi fornecido para este estudo.

\section{REFERÊNCIAS}

1. Hamamoto Filho PT, Venditti VC, Miguel L, Silva LA, Oliveira CC, Peraçoli JC. Pesquisa em educação médica conduzida por estudantes: um ano de experiência do núcleo acadêmico de pesquisa em educação Médica. Rev Bras Educ Med [Internet]. 2011;35(1):108-13.

2. Frantz Krug SB, Assuncao AN, Weigelt LD, Sehnem L, Schmidt Alves LM, Faller $L$ de A. Building Paths, Reporting Experiences: the Trajectory of the Health Studies and Research Group. Texto Context Enferm. $2011 ; 20(4): 818-24$

3. Krahl M, Sobiesiak EF, Poletto DS, Casarin RG, Knopf LA, Carvalho J de, et al. Experiência dos acadêmicos de enfermagem em um grupo de pesquisa. Rev Bras Enferm. 2009;1:146-50.

4. DeFranco DB, Sowa G. The importance of basic science and research training for the next generation of physicians and physician scientists. Mol Endocrinol. 2014;28(12):1919-21.

5. Silva FE. A Avaliação Crítica da Literatura Médica como Instrumento de Complementação Educacional no Internato de Medicina. Rev Bras Educ Médica. $2018 ; 42(1): 25-8$.

6. Masic I, Miokovic M, Muhamedagic B. Evidence Based Medicine - New Approaches and Challenges. Acta Inf Med. $2008 ; 16(4): 219-25$.

7. Aragão JCS, Rossi HR, Casiraghi B. A Jornada do Acadêmico de Medicina - Um Modelo Simbólico da Formação Médica. Rev Bras Educ Médica. 2018;42(1):38-44.

8. Pícoli RP, Domingo ALA, Santos SC dos, Andrade AHG de, Araujo CAF, Kosloski R de MM, et al. Competên- 
cias Propostas no Currículo de medicina: Percepção do Egresso. Rev Bras Educ Médica. 2017;41(3):364-71.

9. Pires-alves FA, Cueto M. The Alma-Ata Decade : the crisis of development and international health. Cien Saude Colet. 2017;22(7):2135-44.

10. Kassab P, Lourenço LG, Ilias EJ, Malheiros CA. A tragédia do ensino médico no Brasil. Rev Assoc Med Bras. 2013;59(4):305-6.

11. Nogueira MA, Canaan MG. Os "iniciados": os bolsistas de iniciação científica e suas trajetórias acadêmicas. Tomo. 2009;(15):41-70.
12. Burgoyne LN, Flynn SO, Boylan GB. Undergraduate medical research: the student perspective. Med Educ Online. $2010 ; 15(5212): 1-11$.

13. Amorim FF, Santana LA, Toledo EF da RJ,Da Silva CCG, Balieiro $V$ de AT, Almeida KJQ de. Undergraduate research in medical education. Rev Assoc Med Bras. 2017;63(12):1017-8.

14. Gadelha CAG, Temporão JG. Development, Innovation and Health : the theoretical and political perspective of the Health Economic-Industrial Complex. Cien Saude Colet. $2018 ; 23(6): 1891-902$. 